\title{
ECG Data Provisioning for Telehomecare Monitoring
}

\author{
Bernardo Gonçalves ${ }^{1}$, José G. Pereira Filho ${ }^{1}$, Rodrigo V. Andreão ${ }^{2}$, Giancarlo Guizzardi ${ }^{1,3}$ \\ ${ }^{1}$ Computer Science Department \\ Federal University of Espírito Santo \\ (UFES), Vitória (ES), Brazil \\ ${ }^{2}$ Electrical Engineering Department \\ Federal University of Espírito Santo \\ (UFES), Vitória (ES), Brazil \\ ${ }^{3}$ Laboratory for Applied Ontology \\ ISTC-CNR \\ rodrigo@ele.ufes.br \\ Trento, Italy \\ \{bgoncalves,zegonc\}@inf.ufes.br \\ guizzardi@loa-cnr.it
}

\begin{abstract}
The latest computer and communication technologies in combination with an enhanced ECG analysis system can be used to improve cardiac patient's follow-up out-of-hospital. In this way, real-time transmission of the so-called ambulatory electrocardiogram (AECG) to a remote health application with awareness of the patient's context can support decision making as well as to allow efficient emergency attendance. However, the effectiveness of such a service requires tackling challenges of hardware and software beyond the common issues normally addressed in the literature. This paper proposes an ECG provisioning system that handles advanced issues such as flexibility and interoperability in pervasive scenarios as much as the well-known need for efficient transmission. This system architecture embraces an ECG analysis system based on hidden markov models and makes use of an original ECG markup language.
\end{abstract}

\section{Categories and Subject Descriptors}

J.3 [Computer Applications]: Life and Medical Sciences- health; D.4.7 [Operating Systems]: Organization and Design- distributed systems, hierarchical design, real-time systems and embedded systems;

\section{General Terms}

Design

\section{Keywords}

Telecardiology, Telehomecare monitoring, ECG data, Contextawareness, Emergency and decision support

\section{INTRODUCTION}

Computer and communication technologies applied for acquiring vital signs are promoting novel modalities of patients' health telemonitoring. As a result, nowadays physicians can follow their patients' health condition in real-time and report diseases remotely. Telecardiology, in particular, has developed itself

Permission to make digital or hard copies of all or part of this work for personal or classroom use is granted without fee provided that copies are not made or distributed for profit or commercial advantage and that copies bear this notice and the full citation on the first page. To copy otherwise, or republish, to post on servers or to redistribute to lists, requires prior specific permission and/or a fee.

SAC'08, March 16-20, 2008, Fortaleza, Ceará, Brazil.

Copyright 2008 ACM 978-1-59593-753-7/08/0003_..\$5.00. mainly through the acquisition and transmission of electrocardiograms (ECG).

Considering that heart diseases are among the major causes of mortality, this sort of service has called a lot of attention in the last years as an object of research [6], [15], [18]. An example of such initiative in Brazil is the TeleCardio project [3]. TeleCardio is a telehomecare system for remote monitoring of patients with cardiological syndromes. TeleCardio takes advantage of mobile and wireless technologies for the acquisition and transmission of the AECG. The key point is that, when combined with a processing system as far as with those technologies, the AECG allows the diagnosis and/or therapeutic treatment of the myocardial ischemia, an outstanding heart disease that characterizes pre-infarcts. Most of the ischemic episodes take place during daily activities (e.g. physical, emotional) and may lead to a heart attack [8]. TeleCardio system then handles patient's heart monitoring throughout his/her daily activities in order to detect and quantify ischemia episodes at real-time allowing for an efficient emergency rescue. This issue is addressed in TeleCardio by taking into account the concept of context-awareness [9], i.e., the healthcare applications which use the ECG data must change their behavior in response to modifications in the patient's context. For example, consider the situation where a physician wishes to be notified with a SMS message if his/her patient has an arrhythmia. Besides the emergency support, TeleCardio also aids physician's decision making.

Basically, these two features are results of an ECG processing method based on the work of Andreão et al. [2] that carries out a classification on the ECG waveform. Nonetheless, the service of context-aware AECG data provisioning in real-time for multiple healthcare client applications is still far from a trivial task. There are many challenges regarding both hardware and software such as mobility for the patient, ECG data representation, minimizing the delay in real-time ECG data delivery, designing for reuse and so on. In order to tackle these issues, in TeleCardio project we have applied a domain engineering approach for developing ECG Wrapper, one of its major components. ECG Wrapper is an integrated TeleCardio sub-system (hardware plus software) committed to ECG data acquisition, processing, wrapping and delivery in real-time.

This paper focuses on the architectural aspects of ECG Wrapper, discussing how it deals with ECG data provisioning issues. We elaborate on the appropriateness of some mobile and wireless technologies for suitably addressing data transmission on both patient's side and physician's side. Besides, we argue that the appliance of an ECG automatic analysis system is purposefulness 
when combined with an ECG data model designed to meet the needs of telemonitoring in the sense of both alarm generation and decision support. We advocate that such an ECG format should comply with not only efficient transmission, but also with flexibility, truthfulness, interoperability over the Internet and between heterogeneous health systems and so on.

This paper is organized as follows. Section 2 briefly presents TeleCardio project; Section 3 elaborates on the ECG Wrapper conceptual architecture; Section 4 presents an example of ECG Wrapper operation based on one of the TeleCardio scenarios; Section 5 discuss some key experiments carried out to verify the functionality of the modules of ECG Wrapper; Section 6 discusses related work; and lastly, Section 7 concludes the paper and presents future work.

\section{THE TELECARDIO PROJECT}

In TeleCardio project we have developed a set of hardware and software elements, which includes: (i) a novel middleware for supporting the development and deployment of mobile and context-aware applications, named Infraware [17]; (ii) the $E C G$ Wrapper, with its original mechanisms for ECG signal analysis and for automatic alert generation; and (iii) an application server that supplies web-based client applications with access to the system features. In this way, TeleCardio system opens the possibility for a wider number of chronic patients to have access to emergency services, through the use of a diversity of contextaware (mobile) applications running on top of it.

The TeleCardio's middleware infrastructure provides a number of basic and suitable context-aware services for supporting the creation of mobile and context-aware applications. These services include: (i) data integration, (ii) subscription and privacy control, (iii) semantic service description, discovery and management, (iv) context interpretation, and so on. A full description of the TeleCardio project and its middleware components falls outside the scope of this paper and can be found elsewhere [3], [17]. Enduser applications take advantage of such features to provide value added services in Healthcare domain. Besides, the middleware also mediates interactions between the ECG Wrapper and the application server. This mediation is made clear in Section 4.

The application server in turn uses the services supplied by the middleware as well as presents the ECG data obtained from the ECG Wrapper to physician users. This ECG data presentation takes place on web-based client applications, i.e., ECG viewer applications, in such a way to exploit the features the TeleCardio system conveys.

\section{THE ECG WRAPPER ARCHITECTURE}

The ECG Wrapper has a layered design, which means that its internal functions are hierarchically organized. Each layer interacts only with adjacent layers, providing services to the upper layer by building upon the services from the lower layer. Indeed, this architecture is an instantiation of the Context Wrapper [14], a generic sensor data provisioning system also developed in the TeleCardio project.

The architecture is composed by the following entities (see Figure 1):

- Sensors Communication layer (SC): receives the ECG signal from the recording device by a communication protocol;

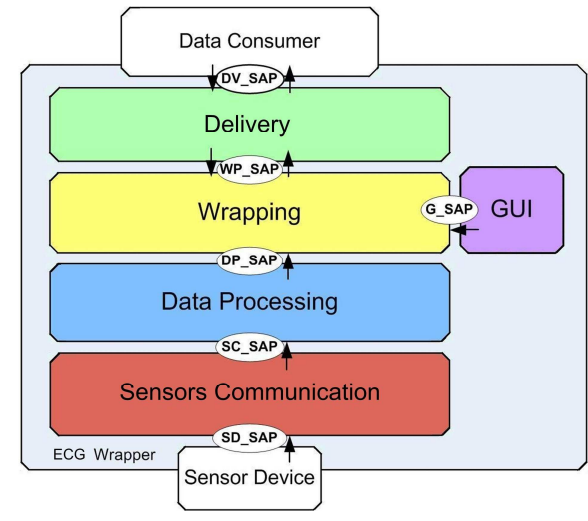

Figure 1. The ECG Wrapper architecture.

- Data Processing layer (DP): carries out ECG data automatic processing to analyze the signal in view of alarm generation and decision support;

- Graphical User Interface (GUI): allows user (the patient or a health agent) to insert patient's personal data and/or electronic patient record (EPR) obtained from anamnesis as well as for recording session parameters configuration;

- Wrapping layer (WP): encapsulates the ECG data units came from DP layer as well as the data obtained from the GUI entity into a feasible format;

- Delivery layer (DV): makes the ECG data available on the web for delivering to health end-user applications and holds subscriptions for the TeleCardio's middleware to take advantage of the services it provides.

The Sensor Device (SD) and Data Consumer (DC) entities interact with ECG Wrapper entities at their boundaries to supply and consume ECG data, respectively. These interactions, as much as the internal interactions, take place at service access points (SAP) through service primitives. In this paper, however, we focus on structural aspects of the system, leaving out a low level behavior description, which is presented elsewhere [14]. Following, we elaborate on each one of the four layers.

\subsection{Sensors Communication (SC) Layer}

The ECG is a continuous signal of low amplitude. It can be digitally acquired by a recording device through $\mathrm{A} / \mathrm{D}$ conversion at a particular sampling rate and assigned to each sample a binary word. However, because of its low amplitude, at least preamplification and pre-filtering stages are implemented at hardware level in the analogical domain. In TeleCardio project we have implemented a portable device (a Holter monitor) that holds such an ECG acquisition, see Figure 2.

Because we are talking about telehomecare, there is no need to embed the ECG Wrapper system into such a device. We rather use a laptop computer where the system takes place. Thus, in order to give mobility for the patient roughly a short covered area in domicile environment, we handle data transmission from the Holter device to the computer over a radiofrequency (RF) wireless link. This communication service is addressed by the SC layer. This service is confirmed and connection-oriented since ECG are time-series and continuous data such that data units only have a 


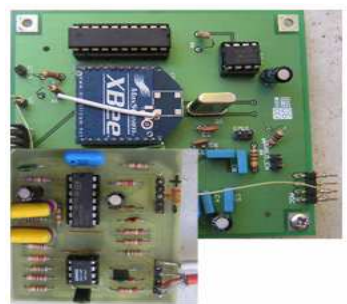

Figure 2. ECG signal acquisition and transmission circuits based on the ZigBee standard.

meaning as a whole. Thus, we ensure that every data unit arrives, orderly, at its destination, i.e., at $S C$ SAP.

To address this SC layer, we have adopted the ZigBee WPAN communication protocol [23]. The ZigBee is based on the IEEE 802.15.4-2003 Low Rate WPAN standard, and takes advantage of the specifications concerning the physical and data link layers of the OSI model, adding then its own specifications for the network, security and application layers.

In fact, rather than other protocols such as Bluetooth [5] or IrDA [16], ZigBee is suitable for monitoring and control. On one hand, Bluetooth is designed for supporting the transmission of a substantial quantity of data (e.g. multimedia data) at high transmission rates and infrared-based protocols (e.g. IrDA) require line-of-sight. On the other hand, ZigBee meets the need for low power consumption and low data transmission over RF link to keep as far as possible the batteries of the devices. The ZigBee also fulfills a need for integrating other vital signs' sensor devices (e.g., blood pressure monitor, oximeter), such as it is the case in further work of the TeleCardio project. That is because ZigBee fits better requirements of wireless sensor network such as reliability, adaptability, latency and scalability [20].

We have configured the ZigBee transmission circuit to send data packets of 30 seconds of ECG for the ECG Wrapper at each 30s. This time window was defined taking into consideration the optimal time interval for handling abnormal events such as missed packets, the initial connection establishment, and the period sufficient for contacting the emergency service.

\subsection{Data Processing (DP) Layer}

This DP layer answers for the ECG automatic analysis in order to detect abnormal events on the patient's heart activity as well as to aid the physician's analysis. In fact, the analysis of the ECG waveform can identify a wide range of heart illnesses, which can be characterized through specific modifications on ECG elementary waveforms. The physician expert uses an approach of three different levels to analyze the ECG: (i) detection of the QRS complex and segmentation of the heart beat; (ii) classification of the beat; and (iii) long-term analysis. ECG analysis systems handle the concerns of these different levels by using techniques such as heuristic, statistic, decision trees and fuzzy logics, hidden Markov models, neural networks, rule-based and so on.

The DP layer then comprises such an ECG analysis system, which is proposed by Andreão et al. [2]. This system function is the processing of the ECG raw data received from the portable device. This work is performed through an ECG segmentation and classification approach based on hidden Markov models (HMM).

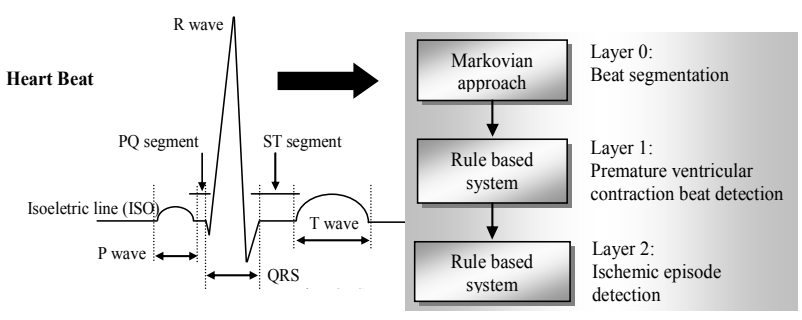

Figure 3. Block diagram of the ECG signal processing.

As Figure 3 shows, the algorithms used in the system (i.e., in DP layer) are organized in a structure composed by three sub-layers.

The sub-layer 0 holds the basic ECG signal processing, which consists in identifying in one ECG record the elementary morphologies necessary to perform any kind of diagnosis. To this end, the ECG signal is processed by the Mexican Hat wavelet transform, which emphasizes signal contents and overlooks the noise. Then, a stochastic approach based on HMM's performs the ECG signal segmentation in terms of the elementary waveforms of the heart beat. In fact, the segmentation strategy associates the processed samples of the ECG signal with the HMM of each beat waveform in order to maximize the likelihood. From the output of sub-layer 0 , the layer 1 classifies each heart beat through a heuristic ruled based system (i.e., beats are classified as normal or premature ventricular contraction), which models the physician approach. Finally, at sub-layer 2, the system carries out a longterm analysis aiming at identifying abnormal events (i.e., ischemic episodes) [2].

The major benefit of such a markovian approach compared with a heuristic one relies on its statistical nature, which allows the adaptation of the models used to the ECG samples analyzed through training [2]. By means of this approach, each ECG data unit, i.e., each packet of $30 \mathrm{~s}$, is processed as far as it has been received. The data units then are passed to the WP layer that encapsulates them into an ECG data model. In case an abnormal event is detected, the ECG data unit is flagged with true for alarm.

\subsection{Wrapping (WP) Layer}

The wrapping procedure is the encapsulation of the ECG timeseries data combined to its classification and related data (e.g., patients' personal data, electronic patient record, his/her context at the recording session, etc.) into an appropriate data model. This model design has to consider that those data shall be deployed in the Internet for heterogeneous data consumers such as web-based applications running either upon traditional computers or PDAs, handheld devices and smartphones, hospital information systems, etc. Thus the model design must take into account the following non-functional requirements: (i) interoperability between heterogeneous systems; (ii) flexibility, for allowing minimal effort in modifications; (iii) lightness of data as quite as possible, for reaching efficient transmission of its instances; (iv) truthfulness, for being consistent with the ECG domain; and (v) readability, for permitting evaluation of ECG domain experts. Indeed, this model is related to an abstract and to a transfer syntax by means of $(i, i i$, $i v$ and v) and (iii) requirements respectively. These two perspectives focus on data representation and data transmission and are addressed in the OSI reference model, for example, by the application and presentation layers. 
We have, in fact, carried out an extensive research on this ECG data modeling issue. As a first result, we have produced an ECG domain ontology [12]. As we advocate in that paper, the existing standards for representing ECG (e.g., AHA/MIT-BIH [11], SCPECG [19], FDADF [7], ecgML [21]) lack partially or totally the issues just mentioned. We thus deemed quite suitable to develop ecgAware [13], an ECG XML-based markup language. The need for a novel ECG markup language is mainly concerned with telemonitoring through the AECG. The former standards do not consider this concern and then would constitute a limiting interface for transmitting the fullness of AECG data classification as well as the patient's context during the recording session. The ecgAware then extends such standards to cover this gap in the literature.

In ECG Wrapper, then, all the information produced in DP layer is wrapped into the ecgAware model (see Figure 4). The ECGStudy element (the root one) on the model comprises a study of one or more ECG records of the patient. A Record element in turn represents the whole ECG waveform acquired from one ECG recording session, which may have duration of up to 48 hours. In TeleCardio real-time transmission, however, this record is broken into small pieces of 30 seconds of data (a unit) to be later (exactly at the end of the session) gathered again. The RecordingSession element holds the updated context of the patient during the session. This information may be, e.g., (i) the activity performed by the patient while his/her heart activity is monitored, (ii) a boolean flag and a timestamp for indicating whether and when an alarm has taken place, (iii) a field for keeping the updated patient's location, and so on.

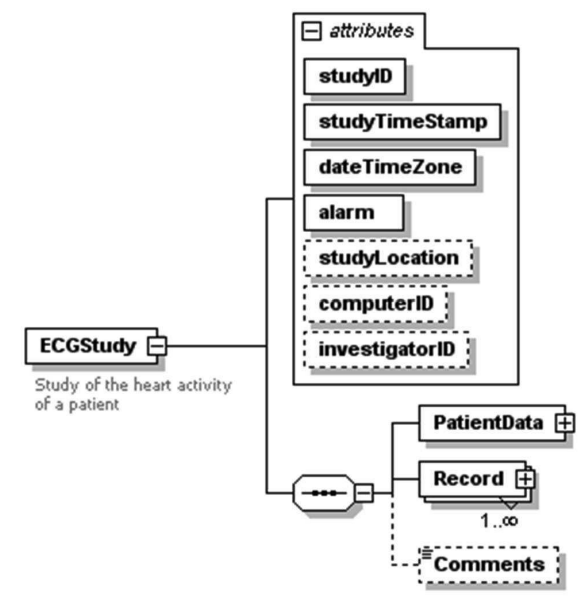

Figure 4. A picture of the ecgAware model.

\subsection{Delivery (DV) Layer}

The ECG data delivery calls for a channel communication (e.g., the Internet, $3 \mathrm{G}$ networks) in order to interconnect the ECG Wrapper (the service provider) and the ECG data consumers in a weak coupled fashion. This non-functional requirement, i.e., weak coupling, remarks itself mostly in pervasive and ubiquitous scenarios where users (in this case physicians or other health professionals) have to access information anytime, anywhere, through applications that require standardized services' interfaces.
That is why in TeleCardio we have adopted the web service (WS) technology [22] for deploying the ECG data in the Internet. The WS technology can promote communication between heterogeneous applications. Once they follow the WS protocols, they are able to access services independently of their platforms and programming languages. Such communication is conveyed over a network using messages and protocols based on XML language as well as on web protocols such as HTTP.

The DV layer thus publishes the ECG data on the web for its identified consumers through a web service. Indeed, the communication with data consumers also takes advantage of the Java remote method invocation (RMI) technology. Whereas RMI is used for performing subscriptions for either Infraware (e.g., log on Infraware) or the application server (e.g., start monitoring), a web service is used for making the data units available on the web. The application server then is able to get and to store each data unit and later to gather them into a record.

The public method the ECG Wrapper deploys on the web is named getEcgData, which is overridden to return either one data unit or a list of data units. This flexibility is useful in case an exception (e.g., missed packets, lost connection) has taken place. In this case, an ECG data consumer might need to get several data units in one single call in order to keep itself updated.

\section{ECG WRAPPER USAGE DESCRIPTION}

Initially (on the installation of the system), a health agent can log in the TeleCardio system through a graphical user interface (GUI) exhibited on the ECG Wrapper system and insert patient's personal data and electronic record data. Both the login data and the patient's register data are transmitted through RMI messages to the Infraware platform that access a user profile database to check and modify information. Then, the user is able to log in TeleCardio as the patient (using his/her account) and to carry out ECG recording sessions. Before starting a session, he/she may provide some data about it. Examples include the expected duration of this session, blood pressure acquired up to that point and so on. Those data are then embedded into the ecgAware model and stored on a XML file. A click on the "Start session" button triggers a RMI message to the health application server communicating such session data and indicating that ECG data units acquired from that patient will be periodically available at a web service interface. The connection between the ECG Wrapper (provider) and the application server (consumer) is based on demand by the consumer, which is able to get each ECG data unit anytime. As previously mentioned, by means of the web public method getEcgData it can obtain either one data unit or a list of them.

However, the start of the session, in fact, is called by the Holter device, which reports to the SC entity that establishes a connection between this device and the system. When the first ECG data unit (a packet of 30s of ECG) has been acquired, the device sends it to the system, which in turn answers by sending a confirmation. At the meantime, the DP entity is listening for the first data unit indication. As far as this indication has taken place, DP entity processes the data and pushes it to the WP entity. This latter then wraps the ECG data unit into the ecgAware model and stores it on a XML file ready for delivery. At this time, the DV entity has already published the web service that is able to deliver the ECG data units which are available. 
Thus, in the normal situation, the availability of a new ECG data unit occurs at each 30 seconds interval (see Section 3.1). However, in case of whether the ECG processing module has detected an abnormal event or the patient has pushed the alarm button of the device, the ECG data unit is flagged true for alarm and then the ECG Wrapper sends a RMI message indicating the emergency situation to Infraware. It in turn contacts the emergency service and sends a SMS message to the physician reporting the alarm. At the end of the session (or by user intervention) the ECG Wrapper sends another RMI message to the application server indicating the session has finished. Then, all ECG data units stored by WP entity are gathered into a single ECG record associated with the whole session.

On the physician's side, a web client of the health application (see Figure 5) allows him/her either to follow-up the recording session in real-time or to access a history of the ECG records available and to view them. This health application takes advantage of the services provided by both ECG Wrapper and Infraware platform to give to the physician his/her patient's condition. The contextawareness of the application relies on that it changes its procedure as a response to some situation which has taken place on the patient's side. The physician then is able to set up options w.r.t. reactive actions (e.g., to trigger a sound alarm on event) and to let her life normally. The GUI interfaces of the application were conceived from fruitful discussions with cardiologists.

\section{EXPERIMENTS}

The ECG Wrapper has been implemented in the context of the TeleCardio project. For now, we have used an ECG simulator device that generates three ECG leads interconnected with our Holter device in order to validate the ECG Wrapper. We have tested the wireless ECG data transmission from the Holter device to the SC layer. Concerning distances of up to 20 meters with physical obstructions, i.e., over the walls, we verified a rate close to $100 \%$ of successful communications carried out at a bit rate of $115,2 \mathrm{kbps}$. Increasing the distance to 30 meters, however, the successful rate has decreased to nearly $40 \%$ at the same bit rate.

The ECG analysis system of the DP layer has been already validated on standard databases and the results are presented elsewhere in [1], [4]: a) our best beat detection results on the QT database is $99,80 \%$ for either sensibility and positive predictivity, while the premature ventricular beat detection rates are nearly $90 \%$; b) for ischemic episode detection, we have tested our system on the European ST-T database, achieving nearly $85 \%$ of either sensibility and positive predictivity. The results above are among the best results already published using the selected databases.

With regard to the WP and DV layers, the delivery of ECG data units (i.e., ecgAware XML files) to the application server has been successful validated. The ECG waveform acquired by the ECG Wrapper is presented online on the ECG viewer. Despite we did not precisely measure the time delay, we are aware that the XML ASCII encoding is an inhibitor to make this data transmission faster. However, a compression procedure of the XML files is a topic of future work in order to achieve a size reduction of such files. In Erfianto's work [10], for example, a reduction of up to $53 \%$ is reached for ECG data and of up to $87,5 \%$ for patient data through parsing the XML Document to an ASN.1 format and in the sequel to a binary-encoded format.

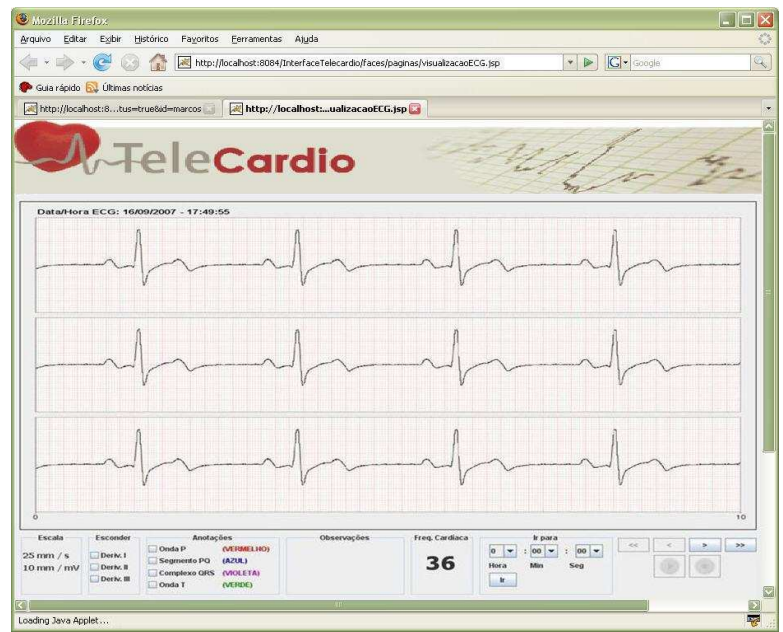

Figure 5. Snapshot of the TeleCardio's ECG viewer.

The real-time visualization of the ECG data transmitted from the ECG Wrapper to the health application has been validated over testing either using the ECG simulated data obtained from the Holter device and using ECG records taken from the MIT database [11]. More robust tests are to be performed by early December 2007 in order to stress the whole TeleCardio system over many real situations, i.e., acquiring the ECG data from patients in recording sessions of $48 \mathrm{~h}$ duration.

\section{RELATED WORK}

There are several initiatives of research regarding Telecardiology for patient follow-up out-of-hospital. Commonly, however, most of the current telehomecare systems offer specific, stand-alone and, sometimes, partial telemonitoring solutions. In the work of Hernández et al. [15], for example, despite the successful achieved with respect to the acquisition, transmission, storage and visualization of the ECG, the authors recognize the need for adding some local machine intelligence to the ECG data acquisition system in order to incorporate ECG analysis, and thus to address alarms detection. Another illustrative case is the Airmed-Cardio project [18], where the system usage, flexibility and interoperability are restricted by the adoption of the SCPECG format. SCP is quite inappropriate for Internet-based communications due to its platform-dependence and hard-coded ECG representation that obstructs experts' evaluation and pushes applications to use it. Moreover, in this work emergency situations are not covered by the system. The patient indeed has to manually send the ECG record at the finishing of the recording session.

\section{CONCLUSIONS \& FUTURE WORK}

The ECG Wrapper system supplies a health application server with ECG data under management of the Infraware platform in the TeleCardio project. We have advocated that ZigBee and Web Services technologies suitably address the SC and DV layers, serving then as communication technologies at the patient's side in a telehomecare system. Another contribution of our proposal is the combination of an ECG automatic analysis system based on hidden Markov models with an ECG markup language designed to meet the needs of telemonitoring in the sense of both alarms 
generation and decision support. Thus, especially by taking advantage of its DP layer, the ECG Wrapper makes TeleCardio an effective proposal for patient's heart telemonitoring. Beyond the decision making support, it is able to detect abnormal cardiac events (e.g., arrhythmias, pre-infarcts, etc.) in order to generate alarms in real-time and then to reduce the response time for rescue. Nonetheless, the usefulness of this DP layer relies on the ecgAware markup language since it preserves the information produced by such an ECG analysis system. In this way, we can foster a value-added telehomecare service for cardiac patients.

The proposed architecture, in fact, tackles some substantial challenges with respect to the diversity of hardware devices and technologies related to ECG data acquisition and usage in telehomecare monitoring. The engineering approach we have adopted in this work has produced a modular integrated hardware and software artifact that allows reuse effortlessly. Future work includes $(i)$ the development of a compression procedure of the XML message produced by the ECG Wrapper; (ii) the incorporation of other scenarios (e.g., an ambulance scenario and an outdoor one) in TeleCardio; (iii) the adaptation of both the health application and the ECG Wrapper for PDA's and handheld PC's for minimizing the size of the devices used as well as lower the price; and (iv) the extension of TeleCardio for other vital signs beyond ECG; and (v) the implementation of a rule based system for abnormality detection based on a statistical approach.

\section{ACKNOWLEDGMENTS}

This research has received financial support from FAPES (grant no. 31024866/2005) and CNPq (grant no. 50.6284/04-2).

\section{REFERENCES}

[1] Andreão, R., et al. ST-segment Analysis Using HMM Beat Segmentation: Application to Ischemia Detection. In Computers in Cardiology, Chicago, 2004.

[2] Andreão, R. et al. ECG Signal Analysis through Hidden Markov Models. IEEE Transactions on Biomedical Engineering, v. 53, n. 8, 2006.

[3] Andreão, R., et al. TeleCardio - Telecardiologia a Serviço de Pacientes Hospitalizados em Domicílio. In X Brazilian Conference in Health Informatics (CBIS'06), Florianópolis, Brazil, 2006.

[4] Andreão, R., and Boudy, J. Combining Wavelet Transform and Hidden Markov Models for ECG Segmentation. EURASIP Journal on Advances in Signal Processing, vol. 2007, 10.1155/2007/56215.

[5] Bluetooth website. http://www.bluetooth.org/, n.d.

[6] Boudy, J., et al. Telemedicine for elderly patient at home: the TelePat project. In Proceedings of the International Conference on Smart Homes and Health Telematics. Belfast, Northern Ireland, 2006.

[7] Brown, B., Kohls, M., and Stockbridge, N. FDA XML Data Format Design Specification, 2002. Available at http://www.cdisc.org/discussions/EGC/FDA_XML_Data_Fo rmat_Design_Specification_DRAFT_B.pdf.
[8] Crawford, M., et al. ACC/AHA Guidelines for Ambulatory Electrocardiography. Journal of the American College of Cardiology, 1999.

[9] Dey, A. K. Providing Architectural Support for Building Context-aware Applications. Ph.D. Thesis, Georgia Institute of Technology, 2000.

[10] Erfianto, B. Design of a Vital Sign Protocol Format Using $X M L$ and ASN.1. M. Sc. Thesis, University of Twente, The Netherlands, 2004.

[11] Goldberger A., et al. PhysioBank, PhysioToolkit, and PhysioNet: Components of a New Research Resource for Complex Physiologic Signals. Circulation 101(23):e215e220, 2000.

[12] Gonçalves, B., et al. An Electrocardiogram (ECG) Domain Ontology. In Proc. of the $2^{\text {nd }}$ Workshop on Ontologies and Metamodels for Software and Data Engineering (WOMSDE'07), João Pessoa, Brazil, 2007.

[13] Gonçalves, B., et al. EcgAware: An ECG Markup Language for Ambulatory Telemonitoring and Decision Making Support. In Proc. of the International Conf. on Health Informatics (HEALTHINF'08), Funchal, Portugal, 2008.

[14] Gonçalves, B., et al. A Service Architecture for Sensor Data Provisioning for Mobile Context-Aware Applications. In Proceedings of the $23^{\text {rd }}$ ACM Symposium on Applied Computing (SAC'08), Fortaleza, Brazil, 2008.

[15] Hernández, A., et al. Real-Time ECG Transmission Via Internet for Nonclinical Applications. IEEE Transactions on Information Technology in Biomedicine, vol. 5, no 3, September, 2001.

[16] IrDA website. http://www.irda.org/, n.d.

[17] Pereira Filho, J. G., et al. Infraware: um Middleware de Suporte a Aplicações Móveis Sensíveis ao Contexto. In Proceeedings of the $24^{\text {th }}$ Brazilian Symposium of Computer Networks, Curitiba, Brazil, 2006.

[18] Salvador, C., et al. Airmed-Cardio: A GSM and Internet Services-Based System for Out-of-Hospital Follow-up of Cardiac Patients. IEEE Transactions on Information Technology in Biomedicine, vol. 9, n. 1, 2005.

[19] Standard Communication Protocol - Computer-assisted Electrocardiography N02-15, 2002. CEN/TC251 Secretariat. Available at http://www.centc251.org/.

[20] Tilak, S., et al. A Taxonomy of Wireless Micro-sensor Network Models. In Proceedings of the ACM Workshop on Wireless Security, ACM Press, pp 28-36, 2002.

[21] Wang, H., et al. A Markup Language for Electrocardiogram Data Acquisition and Analysis (ecgML). BMC Medical Informatics and Decision Making 3:4, 2003.

[22] WS architecture. W3C website: http://www.w3.org/TR/ 2004/NOTE-ws-arch-20040211/\#id226089.

[23] ZigBee Specification. Available at http://www.zigbee.org/. 